\title{
PERIODONTAL STATUS AND TREATMENT NEEDS AMONG THE PALESTINIAN REFUGEES LIVING IN THE UNITED NATIONS CAMPS IN JORDAN
}

\author{
Fouad Houssain M H. AL- Bayati \\ Faculty of Dentistry, University of Malaya, Department of Oral Pathology, Oral Medicine \& Periodontology
}

\begin{abstract}
A periodontal health survey, using the WHO criteria 1997, was conducted among 585 Palestinian refugees living in the United Nations Camps in Jordan, the sample consists of 363 females and 222 males aged 15-54 years. The purposes of this study were to determine the prevalence and the severity of periodontal disease and the treatment needs among this population and to correlate the periodontal status with age and gender. By using WHO periodontal probes, the community periodontal index (CPI), the treatment needs (TN) and the loss of attachment (LA) were measured for subject. The number of permanent teeth loss was also recorded. Females were found to have worse periodontal conditions than males. Also, a significant age difference was present. All subjects of the present population had experienced periodontal disease, where $34 \%$ and $43 \%$ of them had a shallow pocket of $4-5 \mathrm{~mm}$ and deep pocket of $6 \mathrm{~mm}$ or more respectively. The results of this study have shown that 5.48 of sextant per subject in this population were with bleeding or higher, 4.80 with calculus or higher, 2.48 with shallow pockets or higher, and 0.88 with deep pocket. This study demonstrated that the loss of attachment increased significantly with increasing age and $42 \%$ of this population had normal level of periodontal attachment, while the rest of them $(58 \%)$ had different amounts of attachment loss. Almost all subjects of this population needed oral hygiene instruction, scaling and/or removal of overhangs and $43 \%$ of them needed complex periodontal treatment. The mean number of loss permanent teeth was among this population with significant age and gender difference. Our data indicated that high prevalence and severity of periodontal disease were observed in this population require programs for both prevention and treatment. Indonesian Journal of Dentistry 2006; Edisi Khusus KPPIKG XIV:25-3I
\end{abstract}

Key words:

\section{Introduction}

Periodontal diseases are one of the most widespread diseases of mankind. No nation and no region of the world being free from it. ${ }^{i, 2,3}$ This diseases, which is defined as a general term to denote any diseases peculiar to the periodontium parts. Therefore, regardless of the aetiology, is evident in all countries at moderate to high prevalence, results in extensive loss of teeth, so it is considered the most important cause of tooth loss during adulthood ${ }^{4.5}$.

In all surveys where prevalence and severity have been assessed, periodontal diseases has been found to progress throughout life ${ }^{6}$, and the lack of awareness and intervention for treatment of periodontal diseases in adolescents may predispose the individual to advanced periodontal diseases at older ages". 
The figures from the United Nations commissioner for refugees indicate that the number of refugees is more that 17 million-most of them are in Asia. On arrival in a new country, a refuge is faced with a multitude of problems such as different social and cultural climate, change in financial circumstances and adaptation to the new society. Geographic mobility is also frequent. Under such conditions, there is obviously a risk that oral care will have low priority or may be neglected especially when survival is at stake. Also the refugee status could take from few months to several years to ended, so during this waiting period the refugee lives in uncertainty from day to day and in turbulence, and such stress can lead to depression, sadness and lack of self-confidence. Several studies have indicated that refugees should be regarded as groups (at risk) with regard to oral health. However, there are still groups within societies with poor oral health and very utilization of oral health services. Studies have shown that refugee groups belong to this category 7,8 .

Basic oral health surveys provide a sound basis for estimation of the present oral health status of a population and its future for development of national or regional oral health programmes and for planning for appropriate numbers and types of personnel for oral care and will permit comparison with data of other countries, ${ }^{9,10}$ since the lack of reliable baseline data for several oral conditions makes it difficult to set measurable and attainable goals ${ }^{11}$.

From all the above, it was decided to conduct an epidemiological study above the Palestinian refugees concerning the periodontal problems and the requirement for treatment to help the appropriate authorities in planning oral health services and to set goals for the future, since to the best of my knowledge, there is no such data for this population. The aims of the study are:

1- To determine the prevalence and severity of periodontal diseases in the Palestinian refugees living in the united nations camps in Jordan with particular emphasis on differences among different age groups of individuals and between genders.

2- To determine the treatment need of this community according to their periodontal status.

3- To provide baseline data that can help in planning preventive periodontal health programs and for further studies in the future.

\section{Materials and Methods}

The survey was performed according to the basic methods of the oral health surveys of the WHO for the year 1997 and conducted during the period between February to April 2002 in the United Nations camps of the Palestinian refugees in Jordan, after obtaining permission of the concerned authorities. The sample was obtained from the Palesinian refugees living in the United Nations camps in Jordan and attending the social service canters, which are responsible for all refugees, in those camps. This population is a deprived community, with no regular dental care programs. Taking into consideration the age and gender of the population that was involved in this study, the sample size was 585 subjects, whom were randomly selected. The sample ages range between 1554 years, divided into 6 age groups: 15-19, 20-42, $25-29,30-34,35-44$ and $45-54$ years, and the number of subjects in each age groups examined was approximately 90 subjects.

Design of the study. The subjects were examined seated in a chair with a high backrest in a suitable room, with the examiner standing behind or in front of the chair.

Examination was under natural light, so the subjects was positioned so as to receive maximum illumination, while avoiding discomfort from direct sunlight on either the subject or the examiner. The chair was facing an opening through which the light enters, and was placed as close to it as possible. A suitable form for recording the general information and the results of survey described in oral health surveys basic methods of the WHO for the year 1997 was used.

Oral Examination. Examination and oral health assessments were performed according to the basic methods of the oral health survey of the WHO for the years 1997. Periodontal status and treatment needs. By using WHO periodontal probes, the Community Periodontal Index (CPI), the Treatment Needs (TN) and Loss of Attachment (LA) were measured for subject.

Sextants. The mouth is divided into sextants defined by the tooth numbers: $18-14,13-23,24-28$, $38-34,33-43$ and 44-48. a sextants should be examined only if there are two or more teeth present which are not indicated for extraction ${ }^{12}$. Statistical analysis. The data were processed and analysed using the statistical package for social sciences (SPSS Inc., 2000, version 10.1 for windows): 
1-F-test (for homogeneity of variances test).

2-T-test (for statistical analysis of differences for recorded parameters between genders).

3-P-value (0.05 level of significant).

4-Anova test (for statistical analysis of differences among age groups when the homogeneity of variances was present).

5-Kruskal-wallis test (for statistical analysis of differences among age groups when the homogeneity of variances was not present).

\section{Results}

1-Periodontal Status and Treatment Needs A-Community Periodontal Index (CPI). Table 2 shows, the percentage of subjects with gingival bleeding (codel), the prevalence of calculus (code 2) in all age groups. Subjects with shallow pockets of $4-5 \mathrm{~mm}$ (code 3 ) increased from $36 \%$ at the age group (15-19) years to $43 \%$ at the age group (20-24) years then decreased to $38 \%$ at the age group (25-29) years to $31 \%$ at the age group $(30-34)$ and to $27 \%$ at the age groups (35-44) and (45-54) years. On the other hand, the percentage of subjects with deep pockets of $6 \mathrm{~mm}$ or more (code4) increased steadily with increasing age, these differences concerning the highest CPI code recorded per subject among the six age groups were found to be highly significant $(\mathrm{P}<0.001)$.

B.The Mean Number of Sextants with CPI Code (s) Per Subject. As a measure of severity of condition (Table 3) clearly demonstrated that less than one sextants per subject was found to be healthy among the total sample subjects, while the mean numbers of sextants with bleeding or higher, calculus or higher.

C-Gender Differences According to the Highest CPI Code. Table 4 illustrates that females at age group (15-19) years had higher prevalence of bleeding gingival $(7 \%)$ and calculus $(58 \%)$ than females, males showed significant difference compared to females at this age group ( $p<0.05$ ).even it was found that females in each age group except the youngest one had slightly higher percentages of the highest CPI code recorded Per subject. No significant differences were found between both genders in each age group ( $p>0.05$ ), but among the total sample subjects, females differ statically significant from males $\left(p^{<0.05}\right)$.

2-The Treatment Needs (TN).

A-Percentage Distribution of Subjects According to the Type of Periodontal Treatment Needed. Percentages of subjects in each age group with no need for periodontal treatment (TNO) need for oral hygiene instructions (TN1) need for scaling and/or removal of overhangs in addition to oral hygiene instructions (TN2) and need for complex treatment (TN3) are presented in Table 5. It is obvious from Table 5 that no one of the total sample subjects was in no need of periodontal treatment category, while all of them $(100 \%)$ needed oral hygiene instructions and almost all subjects (99\%) needed scaling and /or removal of overhangs in addition to oral hygiene instructions. Forty three percent of the total sample subjects were finally found in need of complex treatment category.

B-Gender Distribution According to the Type of Periodontal Treatment Needed. Table 6 shows that both genders in each age were almost equally distributed according to the first three types of treatment need categories (TNO, TNl, and TN2) except for the age group (15-19) years, where fewer females $(93 \%)$ than males $(100 \%)$ were found in the third treatment need category (TN2), but differences in percentages between genders in each age group were found in the fourth treatment need category (TN3). Among the total sample, it was found clearly again that both genders distributed almost equally on the first three treatment need categories, but on the fourth one females had a higher percentage (47\%) than males $(35 \%)$.

C-Loss of Attachment (LA). A-Distribution of Subjects According to the Highest LA Code Recorded for Each Subject. Table 7 demonstrates clearly that the percentage of subjects with LA code $(0)$ decreased steadily with age. The opposite pictures were found for the prevalence $4-5 \mathrm{~mm}$ loss of attachment (code 1), where it increased from $13 \%$ at the age group (15-19) years to reach its highest level $(51 \%)$ at the age group $(35-44)$, and for the prevalence of $6-8 \mathrm{~mm}$ loss of attachment (code 2 ), where it increased from $2 \%$ at the age group (15-19) and (20-24) years, and reached the highest percentage $(38 \%)$ at the age group (45- 
54) years. For 9-11 mm LA (code 3) and $12 \mathrm{~mm}$ or more LA (code 4), the highest percentage were found at the age group (45-54) years. Differences among all age groups concerning the highest LA code recorded for each subject were statically highly significant $(P<0.001)$.

B-The Mean Number of Sextants with LA Code per Subjects for Each Category. Table 8 show that the mean number of sextants with LA code (0) was the highest among all mean numbers in each age group except for the age group (45-54) years where the mean number of sextant with loss of attachment of $4-5 \mathrm{~mm}$ (codel) was the highest.

C-Gender Differences According to the Highest LA Code. For the total sample and all age groups subjects, no statically significant differences were found between both genders $(\mathrm{P}>0.05)$, as shown in table 9 .

\section{Discussion}

In the present study, we could not base the selection of the sample on a population census lists due to political, social and economical factors. That is because census lists are not avaitable due to the mortality of the population, but our prediction is that there is a higher ratio of females to males because males go early to work and also due to the fact that high numbers of males have been killed and taken as prisoners in different wars that this community faced in the past. For these reasons the present study sample has 363 females $(62 \%)$ and 222 males $(38 \%)$.

\section{Periodontal Status and Treatment Needs.}

1-Community Periodontal Index (CPI):

It is an effective method for measuring and monitoring the magnitude, prevalence and severity of periodontal diseases, as well. its world- wide application allows for international comparisons 13

\section{A-Percentage Distribution of Subjects According} to the Highest CPI code Recorded for Each Subject.

It is clear from the present results that all of the Palestinian refugees in age group had experienced periodontal disease. At the age group (15-19) years, the highest percentage of subjects $(53 \%)$ was found to have calculus. This percentage is somewhat close to those reported from Australia, Germany, Hong Kong, Italy and Niger, but much higher than those reported from France, Japan, the Netherlands, New Zealand, South Africa and USA (14). While at the age group $(20-24)$ years the highest percentage $(43 \%)$ was for those having shallow pockets of 4-5mm and this finding was lower than survey from Egypt, Tanzania and Uruguay, approximately equal to surveys from Brazil, El Salvador, Mongolia, Nigeria and Rwanda, and higher than the other surveys from developing countries reported by. ${ }^{15}$

At the rest of the age groups, the percentage was for those having deep pockets of $6 \mathrm{~mm}$ or more as the worst condition. The percentage at the age groups (25-29), (30-34) and (45-54) years were $41 \%, 60 \%$ and $67 \%$ respectively, and those percentage were higher than those reported from all of the developing countries for the comparable age gropes, at the age group (3544) years, the percentage ( $64 \%$ ) was lower than that reported from developed and developing countries. ${ }^{16}$ It should be noted that the present data indicated that the prevalence of periodontal disease increased statically scientifically with increasing age and this finding is in accordance with numerous other studies. ${ }^{10,11,17}$

Among the total sample subjects, the epidemiological data of the current study showed that none of the Palestinian refugees had a completely healthy periodontal tissue and that this community showed a high prevalence of periodontal diseases. This could be ascribed to the inferior oral hygiene by refugees, who often have more pressing and important problems than their oral hygiene .This present finding is in agreement with findings in many studies among other refugees. ${ }^{7.8}$

B. The Mean of Sextants with CPI Code (s) per Subject

It is clearly demonstrated from the current results that the majority of sextants, over five sextants, among all age groups and the total sample subject were affected by bleeding or higher. Close findings were reported from India, Namibia, Nigeria and Thailand, but our finding was higher than those reported from many other countries, such as Algeria, Brazil, Fiji, Hong Kong, Philippines, Saudi Arabia, Syria, Turkey and Uruguay. 
The mean number of sextants with shallow and deep pockets increased with increasing age in the current results. Also, it should be noted that the severity of shallow pockets per subjects was higher than deep pockets among all age groups and the total sample. So, this community is found to be severely affected by periodontal disease and this finding could be attributed to the inferior oral hygiene status.

C. Gender Differences According the Highest CPI Code

Results in this study have illustrated that the prevalence of periodontal disease at the age group (15-19) years was lower in females than in males and this difference is significant. This is consistent with reports of other authors. ${ }^{18}$ This difference can largely be explained by better oral hygiene among females at this age groups where females become more interested in their appearance. At the rest of the age groups, no significant difference was found between genders, regarding the prevalence of periodontal disease, but among the total sample subjects, females were found to have a significant higher prevalence of periodontal disease than males. This finding could be attributed to the fact that in some developing countries and due to frequent childbirth and poor nutrition, periodontal conditions are worse in females than in males. On the contrary, females were not reported to have worse periodontal conditions than males in many other studies. $^{19,20}$

\section{2 -The Treatment Needs (TN)}

A-Percentage Distribution of Subjects According to the Type of Periodontal Treatment Needed

The results of the present study have shown that none of the subjects in each age groups and the total sample was in no need of periodontal treatment, while almost all of them needed oral hygiene instructions and, and this could be correlated to complete ignorance of their oral hygiene. Regarding complex treatment, the percentage increased from $8 \%$ at the age group (15-19) years, to $23 \%$ at the age group $(20-24)$, to $41 \%$ at the age group (25-29) years, to $60 \%$ at the age group (30-34) years, to $64 \%$ at the age group (35-44) years and reached the highest level $(67 \%)$ at the age group (45-54) years, and among the total sample subjects, the percentage was $43 \%$ and this reflects the massive need of periodontal therapy.
Those figures were higher than that has been previously reported for comparable age groups from Germany in 1988, Japan in 1989, Thailand in 1989, Croatia in 1992, Hong Kong in 1994, Yemen in 1996 and Chile in 1998. Therefore, a priority should be based on population strategy. And organized oral health services have to work on all levels of oral health promotion, education, prevention and therapy of periodontal disease to improve the periodontal health status of the current study population.

\section{B. Gender Distribution According to the Type of Periodontal Treatment Needed}

The epidemiological data of the present study showed a somewhat equal distribution of genders, in each age groups and among the total sample subjects, according to the need of oral hygiene instructions, scaling and/or removal of overhangs, but differences in percentages between genders were found in those who needed complex treatment, where females needed on average more periodontal complex treatment than males. This finding is in disagreement with some earlier studies. ${ }^{\text {T.21 }}$

\section{3-Loss of Attachment (LA):}

A. Distribution of Subjects According to the Highest LA Code Recorded for Each Subject It is demonstrated from our results that the highest percentage of subjects at the age groups (15-19), (20-24) and (25-29) years were for those having normal attachment level. While at the age groups (30-34) and (35-44) years, the highest percentage of subjects were for those having 4-5 mm loss of attachment, and at the age group (45-54)years a higher percentage of those having $6-8 \mathrm{~mm}$ loss of attachment than those in other La categories was found. Our results concerning the prevalence of attachment loss at different age groups were somewhat close to those reported from Hong Kong ${ }^{22}$ and Kenya ${ }^{23}$ and they were different from those results reported China ${ }^{24}$ and Yemen ${ }^{21}$ of the corresponding age groups. Comparisons with other loss of attachment data from other national studies are more difficult because of the differences in methods. It is clear among all the age groups of the present study that the prevalence and the level attachment loss increased significantly with increasing age, and this finding is supported by many authors. ${ }^{2,25,26}$ It is apparent from the present data of the total sample subjects that the loss of attachment is 
highly prevalent in this community. This may be ascribed to the fact that the oral care is given a low priority or many be neglected by refugees.

\section{B. The Mean Number of Sextants with LA Code} per Subject for Each Category

In our study, the highest mean number of sextant per subject was found for those sextants with normal attachment level and lower mean numbers were found for sextant with different levels of attachment loss among each age group except for the oldest one where the highest mean number was found for those sextants with $4-5 \mathrm{~mm}$ loss of attachment. The present results demonstrated that more than two thirds of the sextants $^{4.31}$ per subject were having normal attachment level, while the rest of the sextants were having different levels of attachment loss, where 1.14 of sextants were with $4-5 \mathrm{~mm}$ loss of attachment, less than half sextants $(0.31)$ was with $6-8 \mathrm{~mm}$ LA, 0.08 of sextants was with 9$11 \mathrm{~mm}$ LA and the lowest mean number of sextants (0.01) was for loss of attachment of $12 \mathrm{~mm}$ and more. So, this community is found to be severely affected by loss of periodontal attachment and this finding may be attributed to the inferior oral hygiene status.

C. Gender Differences According to the Highest LA Code. It is revealed from this study that there were no statistically significant differences between females and males in the prevalence of attachment loss among all age groups and the total sample. Similar results were found by some authors ${ }^{27}$, while dissimilar results were found by others ${ }^{28,29,30,31}$ who reported that males have a higher prevalence of attachment loss than females.

\section{Conclusion}

This study has obviously shown that the periodontal disease is highly prevalent in Palestinian refugees, and that the periodontal breakdown increased steadily with age. Periodontal conditions seem to be worse in females than in males in this community, almost all subjects in the present study need oral hygiene instructions, scaling and/or removal of overhangs, and $43 \%$ of them need complex periodontal treatment and this reflects the massive need of periodontal therapy for this community.

\section{Reference}

1- Miyazaki H, Pilot T, Leciercq MH, Barmes De. Profiles of periodontal condition in adults measured by CPINT. Int Dent $J$ 1991b; 41: 74-80.

2- Locker D, Slade GD, Murray H. Epidemiology of periodontal disease among older adults: A review. Periodontol 2000 1988: 16:16-33

3- Buatongsri V, Songpaisan $Y$, Hongprasong $N$. Phantumvanit $P$, Clark $N$. The distribution of severe periodontitis in urban (Bangkok) and rural (payao province) high risk to street group of Thai population. CU Dent J 2002; 25:1-7.

4- Pilot, Miyazaki $H$, Leclercq MH, Barmes DE. Profites periodontal conditions in older age cohorts. measured by CPINT. Int Dent $J 1992 ; 42: 23-30$.

5- Hohlfeld $M$, Bernimoulin Jp. Application of the community periodontal index of treatment needs (CPINT) in a group 45-54-year-old garment factory workers. JClin Perindontol 1993; 20: 551-6.

6- Marshall-Day CD, Stephens RG, Quigley LF. Periodontal disease, prevalence and incidence. Periodontol 1955; 26: 185-203.

7- Zimmerman $M$, Bornstein $R$, Martinsson $T$. Simplified preventive dentistry program for Chitean refugees: Effectiveness of one versus two instructional sessions. Comm Dent Oral Epidemiol 1993; 21: 143-7.

8- Selikowitz HS. Acknowledging cuitural differences in the care of refugees and immigrants. Int Dent $J$ 1994; 44: 59-61.

9. WHO. Oral health surveys basic method. $4^{\text {th }}$ edition, World Health Organization, Geneva, Switzerland, 1997.

10- Pslenstein VWH, Truin GJ, Can N, Khanh ND. The possibility of previous epidemiology data to serve as baseline for future national oral health surveys-a study in Vietnam. Int Dent J 2001; 51: 45-8.

11. Petersen Pe and Kaka $M$. oral health status of children and aduits in the republic of Niger, Africa. Int Dent J 1999; 49:159-64.

12. Ramfjord SP, Massler M, Greene JC, Held AJ, Waerhaug 3. Epidemiology studies of periodontal disease. AJPH 1968; 58: 1713-22.

13- Benigeri $M$, Brodeur JM,Payetee $M$, Charbonneauu A ,Ismail AL. Cmmunity periodontal index of treatment needs and prevalence of periodontal conditions. J Clin Periodontol 2000,27:308- 12 .

14- Pilot T, Barmes DE, Leclercq MH, McCombie BJ, Sardo-Infirri J. Periodontal condition in adolescents, 15-19 years of age, An overview of CPINT data in the WHO global Oral data Bank. Comm. Dent oral Epidemiol 1987; 15:336-8.

15- World Health Organization, Global Oral Data Bank. CPINT data. Developing countries. Geneva: WHO, 2002.

16- World Health Organization, Global Oral Data Bank. CPINT data, age group 35-44 years. Geneva: WHO, 1994. 
17- Beiruti N. Taifour D, Palenstein VWH, Frencken JE, A review of the oral health status in Syria. Int Dent $J$ $2001 ; 51.710$.

18- Harley AF and Floyd PD. Prevalence of juvenile periodontitis in schoolchildren in Lagos, Nigeria. Community Dent Oral Epidemiol 1988; 16:299-301.

19- Gamonal JA, Lopez NJ, Aranda W. periodontal conditions and treatment needs, by CPITN, in the 3544 and 65-74 year-old population in Santiago, Chile. Int Dent $J$ 1998; 48: 96-103.

20- Furuichi Y, Ito Ho, Izumi Y, Matsuyama, Yotsumoto Y,Mishige Y. Periodontal status and serum antibody titers for porphyromonas gingivalis Fimbriae in a rural population in Japan. $J$ Clin Periodonsol 2001; 28: 264-9.

21- Mengel R, Eigenbrodt M, Schunemann T, Tacoby FL. Periodontal status of a subject sample of Yemen. $J$ Clin Periodontol 1996; 23: 437-43.

22- Holmgren CJ, Corbet EF. Periodontal conditions among the middle-aged and the elderly in Hong Kong. Comm. Dent Oral Epidemiol 1994; 22: 396402.

23. Baelum V, Manji F, Wanzala P, Fejerskov $O$. Relationships between CPITN and periodontal attachment loss findings in an adult population. $J$ Clin Periodontol 1995;22: 146-52.

24- Baelum V, Chen X, Manji F, Luan Wm, Fejerskov O Profiles of destructive periodontal disease in different populations. J Periodont Res 1996; 31: 17-26.
25- Kalsbeek H, Truin GJ, Poorterman JHG, Rpssum Gm, Rijikom HM, VErrips GHW. Trends in periodontal status and oral hygiene habits in Dutch adults between 1983 and 1995. Community demt Oral Epidemiol 2000; 28: 112-8

26- Neely Al, Holford Tr, Loe H, Anerud A, Boysen $\mathrm{H}$. the natural history of periodontal disease in man. Risk factors for progression of attachment loss in individuals receiving no oral health care. $J$ Periodontol 2001; 72: 1006-15.

27. Al-safi KA. Periodontal condition in a randomly selected population in Aubed Al-Sahar Village in Rathwania. J College Dent 1999; 5: 73-5.

28- Baelum V, Fejerskov $O$, Manji $F$. periodontal diseases in adult Kenyans. $J$ Clin Periodontol 1988: 15: 445-52.

29. Gilbert GH and Heft MW. Periodontal status of older Floridians attending senior activity centers. $J$ Clin Periodontol 1992; 19: 249-55.

30- Oliver RC. Brown LJ, Loe H. Periodontal disease in the united states population. $J$ periodontol 1998; 69: 269.78.

31- Sbaraglia M, Turnbull RS, Lacker D. Risk indicators for periodontal disease in a remote Canadian community. J Public Health Dent 2002; 62: 51-6.

32- Schurch E, Minder C, Lang NP, Geering AH. Periodontal conditions in a randomly selected population in Switzerland. Comm Dent Oral Epidemiol 1988; 16: 181-6. 